\title{
Crash occurrence probability and stock market efficiency the indie stock exchange case via Shannon entropy
}

\author{
Boubaker Adel \\ adel.boubaker@fsegt.rnu.tn \\ Sahli Lamia (Corresponding author) \\ sahli_lamia@yahoo.fr
}

Received: August 17, 2012 Accepted: October 13, 2012 DOI: 10.5296/ijafr.v2i2.2526

\begin{abstract}
In this study, we evaluate the relationship between efficiency and probability of the crash, thus the evolution of the daily informational efficiency is measured for the indie stock market index. The efficiency, which is the issue addressed by the weak-form efficient market hypothesis, is calculated using a new method the Shannon entropy and the symbolic time series analysis. A logit model is applied in order to study the relationship between efficiency and probability of the financial crash.
\end{abstract}

Keywords: Informational efficiency, financial crashes, the Shannon entropy, logit model 


\section{Introduction}

The theory of efficiency is considered as one of the most controversial theories in the financial markets theory. Despite the abundance of empirical work to test the hypothesis of efficiency, no clear conclusions seemed. This lack of consensus results is probably related to the importance of efficiency in financial theory. A market may be defined as efficient in the informational sense if the prices of the assets traded on that market instantaneously reflect all available information. The weak-form version of the Efficient Markets Hypothesis (EMH) has been subjected to years of rigorous empirical testing across national stock markets. Since Samuelson (1965) introduced the concept of fair game to financial economics, the EMH is still in controversy. Researchers' views on the EMH have changed during the past 40 years. Fama (1970) summarized that, in early researches, many articles supported the EMH; Fama (1991) himself modified his view because of a lot of anomalies reported after Fama (1970). However, the bulk of the literature focused mainly on testing whether the stock market is efficient or inefficient for the selected sample period, using statistical tests such as the serial correlation tests, runs test, variance ratio tests, unit root tests and spectral analysis. Today nobody can summarize the dispute over the EMH in a couple of lines. The battle between proponents of the EMH and advocates of behavioral finance is still ongoing and, the authors believe, will never end. Considering the possibility of finding the deterministic chaos in financial markets, or at least the fractal Brownian motion some authors have proposed the Hurst exponent as a measure of efficiency, Peters [1994.1996]. Grech and Mazur [2004], Taback and cujeiro(2004,2005, 2008, 2009) have employed the Hurst exponent to measure changes in efficiency over time. However, some authors, such as Bassler et al [2006] and Mc Cauley and others [2007] criticize this measure and argue that a Hurst exponent different from $1 / 2$ (the number corresponding to a random walk process does not necessarily imply correlations of long-term as those found in fractional Brownian motion.

In the present work a new methodology suggested in order to measure the efficiency of information, commonly used in information theory. Our research methodology is distinguishable from previous studies in at least three points. First off all the symbolization of returns is applied. Secondly the Shannon entropy is calculated to measure the amount of information contained in the series, The Shannon entropy is larger the market is more efficient. Thirdly, a logit model is applied to study the relationship between efficiency and the probability of financial crash, the logit model suggests that a decrease in efficiency increases the probability of a crisis on the financial market.

The reminder of the paper is organized as follows. In the following section we will present the methodology Measurement of efficiency by the Shannon entropy and the logit model will be explained. In section 2 we will present the results for the BSE index. Finally, we summarize the finding of this paper in the last Section.

\section{Research Methodology}

Risso [2008] supposes that on an efficient market the actual prices (Pt) reflect all the available information. Therefore, we assume a perfectly efficient market, the returns are unpredictable. The model most commonly used to measure the efficiency is the random walk 


\section{Macrothink}

model. This model has been intensively studied by Hurst in 1940s and later by Mandelbort in 1960s and 1970s, Grech and Masure [2004] uses the local Hurst coefficient to measure efficiency. In the present paper instead of the Hurst exponent uses the Shannon entropy.

\subsection{Measuring the informational efficiency by the Shannon entropy}

Firstly, we assume that on an efficient market the actual prices reflect all available information. Therefore, we assume that the returns in efficient market are unpredictable. The measure of efficiency is computed in two steps. First the symbolization of returns is applied in order to detect the changes over time. Secondly, the Shannon entropy used in order to measure the quantity of information contained in the series. Using the Symbolic Time Series Analyses (STSA), we can obtain richer information from a time series if we transform data series of many possible values into a symbol time series of only a few distinct values.

The problem of symbolic analysis is that there is no formal way to define the time series partitions. However, in our case, we are interested in combinations of negative and positive stock market returns. Therefore, we take (0) for negative returns and (1) for positive ones. In fact, in this case the process is a sequence of Bernoulli processes ( $0 \mathrm{~s}$ and $1 \mathrm{~s}$ ).

Thus we define a time series of size $T$ defined as $\left(r_{1}, r_{2}, r_{3}, \ldots, r_{T}\right)$, being $r_{t}$ the asset returns at time $\mathrm{t}$, for $\mathrm{t}=1,2,3 \ldots \mathrm{T}$.

$\mathrm{r}_{\mathrm{t}}=\log \mathrm{P}_{\mathrm{t}}-\log \mathrm{P}_{\mathrm{t}-1}$

Then we proceed to transform the time series in a symbolic one, according to eq (2).

$$
\begin{cases}r_{t}<\mathrm{O} & \mathrm{s}_{\mathrm{t}}=\mathrm{O} \\ r_{\mathrm{t}} \geq \mathrm{O} & \mathrm{s}_{\mathrm{t}}=\mathbf{1}\end{cases}
$$

Thus it is obtained the following symbolic time series $\left(\mathrm{s}_{1}, \mathrm{~s}_{2}, \mathrm{~s}_{3}, \ldots ., \mathrm{s}_{\mathrm{T}}\right)$, it is the same time series, as sequences of $0 \mathrm{~s}$ and $1 \mathrm{~s}$, representing the decreases and increases in prices, respectively.

First, we implement the Shannon entropy $(\mathrm{H})$ as our measure of efficiency. This measure of the making its maximum value at 1 when the process is completely random, and its minimum at 0 , when there is a completely certain event. The theoretical expression for $H$ is the Eq. (3).

$$
H_{t}=\left(\frac{-1}{\log _{2}(n)}\right) \sum p_{t} \log _{2} p_{t}
$$

(3)

Where $\quad \mathrm{n}$ : total number of sequences

Pi: the probability of sequence $i=1,2, \ldots, n$ 


\section{Mll Macrothink}

It must be noted that the entropy reaches its maximum value 1 when for the $\mathrm{n}$ sequences or events are equally probable, $\mathrm{p}_{1}=\mathrm{p}_{2}=\mathrm{p}_{3}=\ldots=\mathrm{p}_{\mathrm{n}}$. However, the entropy reaching its minimum value 0 when one event cumulates all the probability. It means that there is a completely certain event. For example, if we computed our measure for only two events, assuming that $\mathrm{P}$ is the probability of having a decrease in prices (symbol 0) and (1-P) is the probability of having an increase in prices (symbol 1), according to the Shannon entropy the efficiency will be given by equation (4).

$$
H_{t}=\frac{-1}{\log _{2}(2)}\left(p \log _{2} p+(1-p) \log _{2}(1-p)\right)
$$

(4)

We should mention that $\mathrm{H}$ is a concave function, its maximum value is obtained for $\mathrm{p}=1 / 2$ and its minimum value is obtained for $\mathrm{p}=1$ and $\mathrm{p}=0$. In practice, the probability of decrease and increase in price is the frequency of each event throughout the period divided by the total number of events in the same period. Since we are interested in the evolution of efficiency over time, we take a sub-period $\mathrm{V}<\mathrm{T}$ and move it through time. Then, the entropy is computed for each time window from moment 1to T. Different window sizes are considered, 150, 270, 400 days. Grech and Mazur [2004], (using the Hurst exponent) suggest that the window should not be so large in order to capture the locality. We choose the window that adopts the best $\mathrm{R}^{2}$ in the logit model which we employed later to investigate the relationship between efficiency and the probability of financial crises.

\subsection{The logit model}

Logit and probit models are two families' models for binary endogenous variables. Suppose there is a variable $\mathrm{Y}$ that takes one of the two values, 0 and 1 . In the present case, the financial crash (1) and no-crash (0). Let $\mathrm{Y} *$ a latent variable such that:

$$
y_{t}^{*}=\alpha+\beta H_{t}+\varepsilon_{t}
$$

Where $\mathrm{H}$ is the measurement

of efficiency and $\varepsilon_{t}$ follows is called an extreme value distribution, see McFadden [1984]. We do not observe $\mathrm{y}^{*}$ there, but rather $y$, which takes on values of 0 or 1 according to the following rule

$$
y_{t}= \begin{cases}1 & \text { if } \mathrm{y}_{\mathrm{t}}^{*}>0 \\ 0 & \text { otherwise }\end{cases}
$$

(6)

In our study, the variable y takes the value (1) when the empirical distribution of index returns cumulates the $1 \%$ (the negative tail), and (0) otherwise.

According to Johnston and DiNardo [1997], the logit model is given by:

$$
p\left(y_{t}=1\right)=\frac{\exp \left(\alpha+\beta H_{t}\right)}{1-\exp \left(\alpha+\beta H_{t}\right)}
$$




\section{Macrothink}

(7)

Equation (7) indicates that the probability of financial crash in one day, $\mathrm{p}\left(\mathrm{y}_{\mathrm{t}}=1\right)$ depends on the efficiency level $\mathrm{H}$.

The formulation of the model (7) ensures that the predicted probabilities are between 0 and 1. However, the calculation of the probability of change is not as simple as it was in a linear model. The derivative of the probability of crash with respect to the efficiency $\mathrm{H}$ varies with $\mathrm{H}$ as regards the efficiency

varies with $\mathrm{H}$.

$$
\frac{\partial E(y)}{\partial H}=\frac{\exp (\alpha+\beta H)}{(1-\exp (\alpha+\beta H))^{2}} \beta
$$

The logit model can be expressed as the odds-ratio as in equation (9) which is usually more intuitive.

$$
\frac{p\left(y_{t}=1\right)}{1-p\left(y_{t}=1\right.}=\exp \left(\alpha+\beta H_{t}\right)
$$

$\exp \left(\alpha+\beta H_{t}\right)$ is the effect of the independent variable (our measure of efficiency) in the odds-ratio.

\section{Empirical results for indie market}

In this section the previous methodology (Risso, 2008) is used to study indie markets to find the relationship between the efficiency and the different crashes occurred in the past. We take time windows (v) for 150, 270 and 400 days.

\subsection{Descriptive statistics:}

Tables 1 provide descriptive statistics for different market, mean, standard deviation, skweness, kurtosis and the value of statistical normality test of Jarque-Bera. Remember that for a normal law, the skweness is zero and the kurtosis is 3 , the law being characterized by its symmetry with respect to the average and the low probability of extreme points. Under the null hypothesis of normality, the JB statistic follows a Law Chi-square with two degrees of freedom.

Table 1

The descriptive statistics of returns

\begin{tabular}{|l|l|l|l|l|l|}
\hline & Mean & S.D & Skweness & Kurtosis & Jarque-Bera \\
\hline Inde & 0,0013 & 0,0659 & $-0,0507$ & 2,9785 & 5,991 \\
\hline
\end{tabular}

Statistics provided by Eviews 6.0 
As it is shown in table 1 the null normality hypothesis is rejected. Firstly, we see that the coefficient of Kurtosis is very high; it is less than 3. This defect confirms the platykurtic characteristic of the stock market return series. Secondly, the coefficient of Skweness is different from 0; we observe that the coefficient of Skweness is negative for the return of the Shanghai composite index in all the windows. This shows that the distribution of series is unfolded to the left. This indicates the presence of asymmetry, which may be an indicator of nonlinearity, since it is known that Gaussian linear models are necessarily symmetric.

\subsection{The results of the logit model:}

The logit model was estimated using the efficiency measure as an independent variable, taking different order or combination of days $(1,2,3)$ and different time windows (150, 270,400 days). 9 models were estimated in total. The maximum pseudo- $\mathrm{R}^{2}$ is shown in bold characters and highlights the logit model specification which fits the best.

\subsubsection{The Indie stock market index (BSE 30)}

As the first stock exchange in Indie, the Bombay Stock Exchange is considered to have played a very important role in the development of the country's capital markets. The Bombay Stock Exchange is the largest of 22 exchanges in India, with over 6,000 listed companies. It is also the fifth largest exchange in the world, with market capitalization of $\$ 466$ billion.

The Bombay Stock Exchange uses the BSE Sensex, an index of 30 large, developed BSE stocks. This index gives a measure of the overall performance of the Bombay Stock Exchange, and is closely followed around the world. Based on the Sensex, the BSE equity market has grown significantly since 1990

Table 2: $\mathrm{R}^{2}$ index BSE 30

\begin{tabular}{|l|l|l|l|}
\hline & 150 & 270 & 400 \\
\hline 1 & 0.0017 & 0.0008 & $\mathbf{0 . 0 0 3 8}$ \\
\hline 2 & 0.0011 & 0.0002 & 0.0026 \\
\hline 3 & 0.0014 & 0.0001 & 0.0025 \\
\hline
\end{tabular}

We observe that the window $(\mathrm{v}=400,1 \mathrm{~d})$ corresponds to the highest $\mathrm{R}^{2}$. Therefore it is the window that best explains the relationship of the logit model; the exogenous variable $\mathrm{H}$ explains the endogenous variable y. Note also that when we take a larger time-window the fit is worse. The latter happens because at the time-window increases in size, the entropy loses its locality, Grech and Mazur (2004).

The results of the logit model for the window $(\mathrm{v}=400,1 \mathrm{~d})$ are presented in Table 4 and Figure 1: 
Table 3

The logit model estimating results for the BSE 30 index, for windows $(v=400,1 \mathrm{~d})$

Number of obs $=2686$

$\operatorname{LR} \operatorname{chi} 2(1) \quad=\quad 10.18$

Prob $>$ chi2 $=0.0014^{\mathrm{a}}$

Pseudo R2 $=0.0038$

Log likelihood $=-1346.9945$

\begin{tabular}{lllcc}
\hline Crash probc $^{c}$ & Coef. & Std. Err. & $\mathrm{t}$ & $\mathrm{P}>|\mathrm{t}|$ \\
\hline Entropy $\beta$ & -18.68422 & 5.5456 & -3.37 & 0.001 \\
Constant $\alpha$ & 17.45223 & 5.588 & 3.12 & 0.002 \\
\hline Crash probd $^{\mathrm{d}}$ & Odds Ratio & Std. Err. & $\mathrm{t}$ & $\mathrm{P}>|\mathrm{t}|$ \\
Entropy & $3.80 \mathrm{e}+07$ & $2.12 \mathrm{e}+08$ & 3.12 & $0.002^{\mathrm{b}}$
\end{tabular}

The results are obtained by the econometric software STATA.

a: indicates that the model is significant at the $5 \%$.

b: indicates that the coefficients are significant at the $5 \%$.

c: the estimation of equation (7).

d: the model expressed in odds-ratio equation (8).

We should mention that the probability of a crash depends negatively on our measure of informational efficiency $\mathrm{H}$. When the informational efficiency decreases, the probability of having a crisis increase 


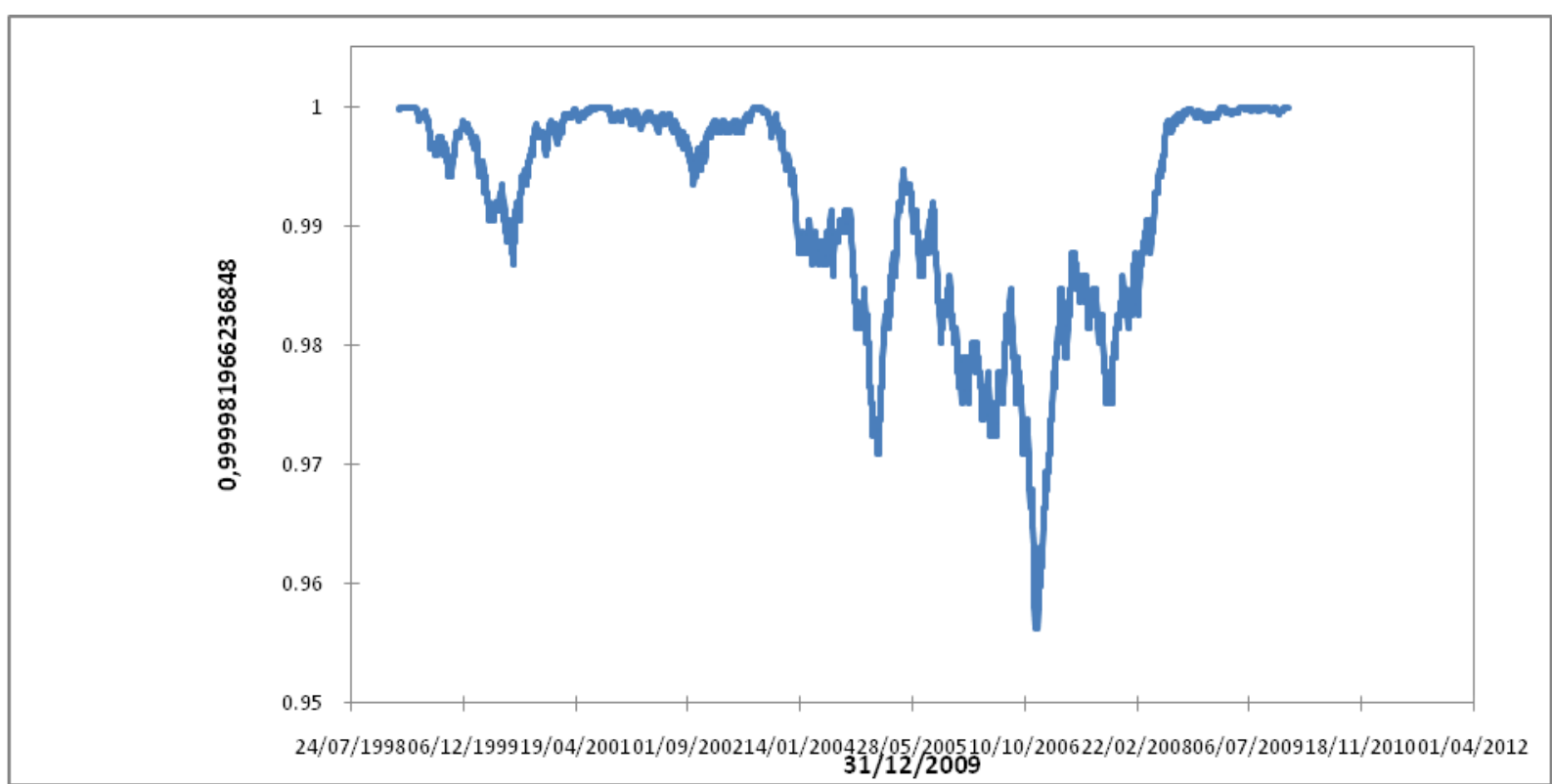

Figure 1: the daily changes of efficiency for the index BSE 30 (inde)

Fig. 1 reports the evolution of the efficiency of the BSE 30 index (taking $v=400$ and an order of 1 day). We note that the numbers of spades inefficiencies are very important between 2004 and 2008. Prior to 2004 the number of stings is very low. The pike October 8, 2007 is the result of international crisis subprime. The collapses of stock prices due to crisis "subprime" mortgage loans at risk. Some analysts talk of economic downturns; others mention a stock market crash. The United States is behind this crisis. Knowing the importance of economy and finance in the U.S. remains the world, we should not be surprised if the consequences are felt everywhere.

\section{Conclusion}

In this paper, a new approach was proposed in order to measure the efficiency of information, commonly used in information theory. Under this methodology the degree of efficiency is done in two stages, first, the symbolization of returns is applied, thereafter, the Shannon entropy is computed to measure the amount of information contained in the series, the larger the Shannon entropy is, the more efficient market is. Once the efficiency is measured, a logit model is applied to study the relationship between efficiency and the likelihood of financial crash, the logit model suggests that a decrease in efficiency produces an increase in the probability of a crisis on the financial market.

The study reveals that the indie financial market tends towards tends efficiency without entirely meeting, so we see that the market goes through periods of inefficiency because of the crisis affecting the international financial market is causing the indie beams of inefficiencies. Moreover our results confirm the negative relationship between efficiency and the likelihood of financial crises, but the market will find the way back to efficiency. 


\section{NI Macrothink}

International Journal of Accounting and Financial Reporting

ISSN 2162-3082 2012, Vol. 2, No. 2

\section{References}

-Cajueiro, D. O. and Tabak, B. M. [2004], "The Hurst's exponent over time: testing the assertion that emerging markets are becoming more efficient"; Physica A; Pages 336, 521; (2004).

- Cajueiro, D. O. and Tabak, B. M. [2005], “Testing for timevarying long-range dependence in volatility for emerging markets"; Physica A, 346, Pages 577-588 (2005).

- Cajueiro, D. O. and Tabak, B. M. [2008], "Testing for time-varying long-range dependence in real estate equity returns"; Chaos, Solitons \& Fractals, Volume 38, Issue 1, October 2008, Pages 293-307

- Cajueiro, D. O. and Tabak, B. M. [2009], "Testing for long-range dependence in the Brazilian term structure of interest rates"; Chaos, Solitons \& Fractals, Volume 40, Issue 4, 30 May 2009, Pages 1559-1573.

- Fama E.F. [1965], "the behavior of stock market prices", journal of Business, vol.38, n¹, Pages 34-105.

-Grech and Z. Mazur [2004], "Can one make any crash prediction in finance using the local Hurst exponent idea? Phys. A336, Pages 133-145.

- Jensen-[1978], "Some anomalous evidence regarding market efficiency", Journal of Finance. Econ. Vol.6, Pages 95-101.

-Johnston J, DiNardo J. [1997], "Econometric methods," fourth ed.McGraw Hill.

-McCauley J, Gunaratne and Bassler G K, [2007] "Hurst exponents, Markov processes, and fractional Browiam motion", Phy. A 379 No. 1, Pages 1-9.

- McFadden - [1984], "Econometric analysis of qualitative choice models. In: Giliches and Intriligator (Eds.), Handbook of Econometrics, North-Holland (Chapter 24).

- Peters. E(1994), "Fractal Market Analysis", First Edition ed.wiley finance.

- Peters. E (1996), "Chaos and order in the capital markets" second ed. Wiley Finance Edition.

- Philippe Gillet, [1999], efficience de marché financier, Edition Economica

- Risso W. [2008], "The Informational Efficiency and the Financial Crashes," Research in International Business and Finance, Vol. 22, Pages 396-408

- Robert Gillet, [1991]," efficience informationnelle du marché boursier: definitions, tests empiriques et interpretation cohérente des resultants", cahiers Economiques de Bruxcelles, $\mathrm{N}^{\circ} 1324^{\text {éme }}$ trimestre 1991.

- Singal [2004], "Beyond the random walk: a guide to stock market anomalies and low-risk investing. Oxford university press.

- Tabak B. M. and Cajueiro D. O., [2006], "Assessing inefficiency in euro bilateral exchange rates", Physica A: Statistical Mechanics and its Applications, Volume 367, 15 July 2006, Pages 319-327 


\section{Macrothink}

International Journal of Accounting and Financial Reporting

ISSN 2162-3082 2012, Vol. 2, No. 2

- Tabak B. M. and Cajueiro D. O.; [2007], "Are the crude oil markets becoming weakly efficient over time? A test for time-varying long-range dependence in prices and volatility"; Energy Economics, Volume 29, Issue 1, January 2007, Pages 28-36 